Letters to the Editor

\title{
Transfusion-Related Acute Lung Injury
}

\author{
Fazal I Raziq, MD \\ Internal Medicine, Michigan State University, USA
}

Article history

Received: 09-04-2020

Revised: 24-05-2020

Accepted: 08-06-2020

Email: iraziq.faza1462@gmail.com

\begin{abstract}
We present here a 50-year-old male with a past medical history of aplastic anemia secondary to myelodysplastic syndrome who presented to the hospital with the chief complaint of severe generalized weakness due to acute anemia. He was transfused one unit of blood and developed ARDS 4 hours later. He was diagnosed with transfusion-related acute lung injury and discharged home after symptomatic management. This case report is a typical case of TRALI and deserves to be published in order to inform the general public of clinicians about TRALI, with the goal to improve the notification rate to hemovigilance which remains very low.
\end{abstract}

Keywords: TRALI, TACO, ALI

\section{Introduction}

Transfusion-Related Acute Lung Injury (TRALI) is characterized by sudden acute respiratory distress following transfusion. It is defined as new, Acute Lung Injury (ALI) occurring during or within $6 \mathrm{~h}$ after blood product administration with no identifiable cause of acute lung injury. All plasma-containing blood products have been reported to cause TRALI (Politis et al., 2016).

TRALI patients present with the rapid onset of dyspnea and tachypnea. There may be associated fever, cyanosis and hypotension. Clinical examination reveals hypoxic respiratory distress without signs of congestive heart failure or volume overload. Chest X-Ray (CXR) shows bilateral pulmonary edema, with bilateral patchy infiltrates, which may rapidly progress to complete "white out" indistinguishable from Acute Respiratory Distress Syndrome (ARDS). Echocardiogram should be obtained to rule out CHF. Some patients exhibit transient neutropenia and a low level of brain natriuretic peptide. However, Neutrophilia is more common. Other laboratory findings include sudden fall in serum albumin as the pulmonary edema in TRALI has high albumin content in analysis (Lieberman et al., 2014; Toy et al., 2012; FRFBCT, 2016). Transfusion-Related Acute Lung Injury (TRALI) may be a lethal adverse reaction in 5 to $15 \%$ of cases. With appropriate care, respiratory status returns to pre-transfusion levels within 48 to $96 \mathrm{~h}$ and CXR returns to normal within $96 \mathrm{~h}$.

We present here a 50-Year-old Male with a past medical history of aplastic anemia secondary to myelodysplastic syndrome who presented to the hospital with the chief complaint of severe generalized weakness and shortness of breath on exertion. On presentation, the patient's temperature was $98.7^{\circ} \mathrm{F}\left[97.8-99.1^{\circ} \mathrm{F}\right]$, heart rate $102 / \mathrm{min}[60-100 / \mathrm{min}]$, respiratory rate $24 / \mathrm{min}$ [12$18 / \mathrm{min}$ ], blood pressure 140/81 $\mathrm{mmHg}$ [90/60-120/80 $\mathrm{mmHg}$, and $\mathrm{SpO}_{2} 98 \%$ [95-99\%] on room air. Laboratory tests were significant for a Hemoglobin of $4.5 \mathrm{mg} / \mathrm{dL}$ (normal 12-15 mg/dL). Chest X Ray showed no acute pathology. Figure 1 All the infectious workup was negative. The patient was admitted to the hospital and 2 units of Packed Red Blood Cells (PRBCs) were ordered. $4 \mathrm{~h}$ after the completion of the first unit of PRBCs, the patient became more tachypneic with a respiratory rate of 34 and $\mathrm{SpO}_{2}$ fell down to $83 \%$. The transfusion of 2nd unit of PRBCs was cancelled. ABG was ordered STAT which showed a PH of 7.50, $\mathrm{PaO}_{2} 45$, $\mathrm{PaCO}_{2} 30$ on room air with a $\mathrm{PaO}_{2} / \mathrm{FiO}_{2}$ ratio of 214 . The patient was put on 12 liters of oxygen by high-flow nasal canula to maintain the saturation at $92 \%$ and the patient was transferred to a step-down unit. Blood pressure was 130/85. At this point STAT CXR was obtained which showed bilateral diffuse alveolar infiltrates. Figure 2 at this point hemolytic transfusion reaction was suspected based on acuity of patient's worsening respiratory status. He denied any rashes. Chest exam was negative for any wheezing or bronchospasm. Blood was drawn and sent to lab for cross match again which ruled out an $\mathrm{ABO}$ or $\mathrm{Rh}$ factor incompatibility. LDH, serum haptoglobin were negative. Urine was sent which was negative for hemoglobinuria. The patient's renal function tests were within normal limits. This ruled out a hemolytic transfusion reaction. At this point our suspicion switched to a myocardial infarction causing acute heart failure. Normal EKG, troponins, BNP levels, Echocardiogram and Central venous pressure ruled out a cardiovascular cause leading to acute volume overload. D dimer level was normal. 


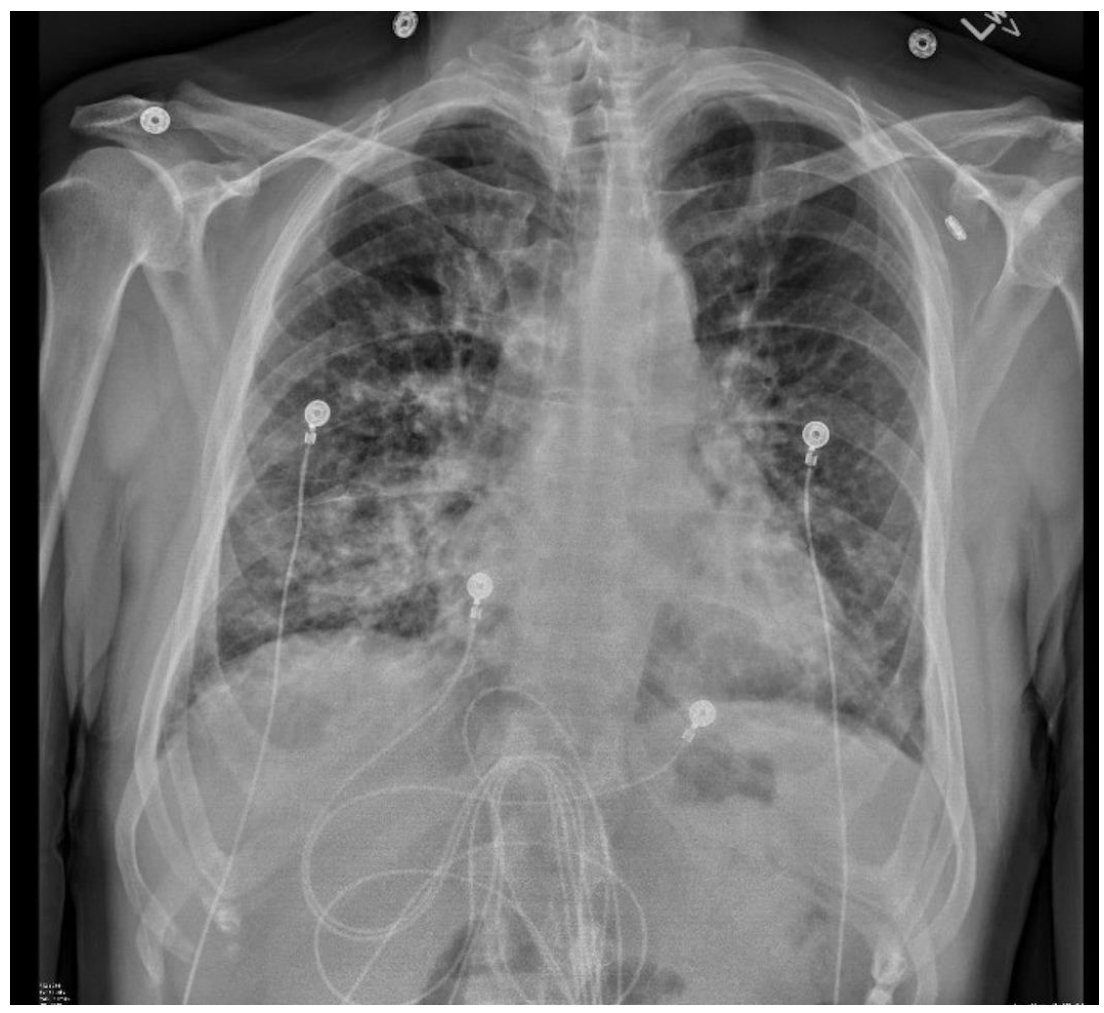

Fig. 1: CXR prior to transfusion does not show any alveolar infiltrate

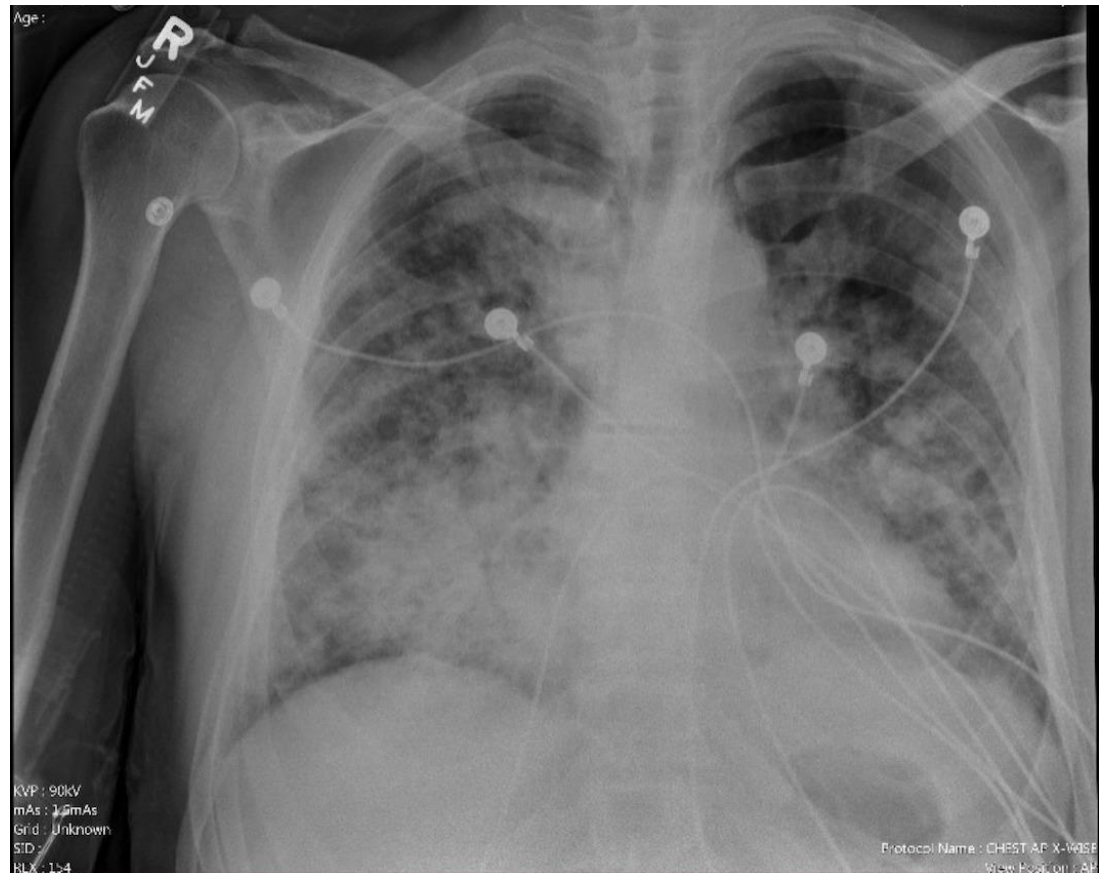

Fig. 2: CXR $4 \mathrm{~h}$ after the transfusion showed Bilateral Alveolar infiltrates

The patient did not have any history or current risk factors for deep vein thrombosis, hence a CTA was not obtained. Blood cultures and peripheral smear was sent with antibody panel as well which included anti
HLA-1 and HLA-2, anti-granulocyte, anti-monocyte and anti IgA. At this point we concluded that the patient's respiratory distress was secondary to Transfusion Related Acute Lung Injury (TRALI) type 1. The blood 
bank was informed about the transfusion reaction. Pulmonology was consulted who recommended supportive care. The patient was kept on the high flow nasal cannula with CPAP at night for the first $48 \mathrm{~h}$ and was subsequently slowly weaned off the oxygen in the following 2 days. On day 5 of admission, the patient was discharged home with outpatient follow up with hematology/oncology. However, the patient was lost to follow up. All the infectious and autoimmune workup came back to be negative ruling out sepsis as the cause of respiratory distress.

The differential diagnosis of acute respiratory distress following a blood product transfusion includes TRALI, Transfusion-Associated Circulatory Overload (TACO), cardiogenic pulmonary edema, allergic and anaphylactic transfusion reactions, TransfusionAssociated Dyspnea (TAD) and bacteremia/sepsis (TTISS, 2012). TRALI may be distinguished from TACO and cardiogenic pulmonary edema by the absence of signs of circulatory overload such as a normal Central Venous Pressure (CVP) and normal Pulmonary Capillary Wedge Pressure (PCWP). Clinical response to diuretics also suggests a diagnosis of TACO rather than TRALI (Semple et al., 2019). Allergic and anaphylactic transfusion reactions may manifest as hypotension and respiratory distress but are marked by laryngeal edema or bronchospasm with wheezing and a normal CXR. An urticarial rash may be present. TAD is acute respiratory distress occurring within $24 \mathrm{~h}$ of transfusion which fails to meet criteria for TRALI, TACO or anaphylactic transfusion reaction. Transfusion-transmitted bacteremia may present with fever and rigors with or without hypotension and culminate in severe sepsis with associated ALI which may be difficult to distinguish from TRALI. The presence of positive blood cultures with the same organism cultured from the implicated blood product is a useful delineating finding.

In order to correctly and consistently diagnose TRALI, the following information must be included in the adverse reaction report:
- Timing of transfusion with respect to symptom onset $(<6 \mathrm{~h})$

- Presence of other risk factors for acute lung injury

- CXR findings suggestive of TRALI

- Evidence of hypoxia: $\mathrm{PaO}_{2}$ or $\mathrm{SaO}_{2}, \mathrm{PaO}_{2} / \mathrm{FiO}_{2}$ ratio of $<300$,

- Clinical indicators of volume status such as clinical evaluation, response to diuretics (if given), or where available Jugular Venous Pressure (JVP), PCWP, CVP, echocardiogram report (Chapman et al., 2009; Silliman and McLaughlin, 2006; Silliman et al., 2005; Bux, 2005; Shander and Popovsky, 2005)

To benefit hemovigilance systems, a Delphi panel in 2019 proposed a modified classification scheme to 2004 Canadian Consensus Conference (CCC) for TRALI (Vlaar et al., 2019). They reaffirmed that TRALI remains a clinical diagnosis and does not require detection of cognate leukocyte antibodies.

The main modifications are presented in the Table 1.

Estimated incidence of TRALI is approximately 1 in 5000 transfusions (Wallis, 2003). As TRALI is a clinical diagnosis its actual incidence is very difficult to predict. Most dedicated clinical studies done in hospitals show that its actual incidence is 4 to 10 times greater than the notification rate (Finlay et al., 2005; Toy et al., 2012).

The reported mortality rate in various publications is between $5-10 \%$. TRALI was the leading cause of transfusion related mortality in the United States until 2016 when it was overtaken by TACO. It is however still the leading cause of transfusion related mortality in the UK (Wallis, 2003; Popovsky and Moore, 1985). In the UK and USA multiple strategies have been suggested to prevent TRALI, which include excluding high risk HLA/HNA antibodies from blood products. Employing this strategy, the reported incidence of TRALI appears to have decreased. However, it's most likely secondary to poor notification rates due to observer bias. In fact, no measures have been taken to prevent TRALI in the HLA/HNA negative donors (Robillard et al., 2007).

Table 1: New TRALI definitions 2019

\begin{tabular}{ll}
\hline Category & Definition \\
\hline TRALI & \\
TRALI Type 1 & No ARDS risk factors and all the criteria given below are met: \\
& A: Acute onset, Hypoxemia with $\mathrm{PaO}_{2} / \mathrm{FiO}_{2}$ ratio of <300, evidence on imaging, No Left atrial hypertension \\
& LAH, or if present not contributing \\
& B: Onset during or <6 h after transfusion \\
& C: No temporal relationship to other risk factors of ARDS \\
& Risk factors of ARDS are present (but ARDS not diagnosed), if mild ARDS is already present it should \\
TRALI type 2 & acutely worsen based on temporal relationship with transfusion based on both of the following: \\
& B: Finding described in TRALI type 1 categories A and B \\
&
\end{tabular}


This case report is a typical case of TRALI and deserves to be published in order to inform the general public of clinicians about TRALI, with the goal to improve the notification rate to hemovigilance which remains very low.

\section{Learning Points/Take Home Messages 3-5 Bullet Points}

1. TRALI may be distinguished from TACO and cardiogenic pulmonary edema by the absence of signs of circulatory overload such as a normal Central Venous Pressure (CVP) and normal Pulmonary Capillary Wedge Pressure (PCWP)

2. TRALI is underreported to hemovigilance networks and clinicians should investigate its possibility in any respiratory distress in the context of transfusion

\section{Acknowledgement}

The author is thankful to the Staff of Michigan State University Internal Medicine residency program for their support and encouragement.

\section{Ethics}

The author re-affirms that publication of this case report follows all the ethical guidelines.

\section{References}

Bux, J., 2005. Transfusion-Related Acute Lung Injury (TRALI): A serious adverse event of blood transfusion. Vox Sang, 89: 1-10. DOI: 10.1111/j.1423-0410.2005.00648.x

Chapman, C.E., D. Stainsby, H. Jones, E. Love and E. Massey et al., 2009. Serious hazards of transfusion steering g. Ten years of hemovigilance reports of transfusion-related acute lung injury in the United Kingdom and the impact of preferential use of male donor plasma. Transfusion, 49: 440-52. DOI: 10.1111/j.1537-2995.2008.01948.x

Finlay, H.E., L. Cassorla, J. Feiner and P. Toy, 2005. Designing and testing a computer-based screening system for transfusion-related acute lung injury. Am. J. Clin. Pathol., 124: 601-601. DOI: 10.1309/1XKQKFF83CBU4D6H

FRFBCT, 2016. Annual summary for fiscal year fatalities reported to FDA following blood collection and transfusion.

Lieberman, L., T. Petraszko, Q.L. Yi, B. Hannach and R. Skeate, 2014. Transfusion-related lung injury in children: a case series and review of the literature. Transfusion, 54: 57-64. DOI: 10.1111/trf.12249
Politis, C., J.C. Wiersum, C. Richardson, P. Robillard and J. Jorgensen et al., 2016. The international haemovigilance network database for the surveillance of adverse reactions and events in donors and recipients of blood components: Technical issues and results. Vox Sang, 111: 409-17. DOI: 10.1111/vox.12447

Popovsky, M.A. and S.B. Moore, 1985. Diagnostic and pathogenetic considerations in transfusion-related acute lung injury. Transfusion, 25: 573-577.

DOI: 10.1046/j.1537-2995.1985.25686071434.x

Robillard, P., C. Hyson and N. McCombie, 2007. TRALI, possible TRALI and respiratory complications of transfusion reported to the Canadian transfusion transmitted injuries surveillance system. Transfusion, 47: 5A-53.

Semple, J.W., J. Rebetz and R. Kapur, 2019. Transfusionassociated circulatory overload and transfusion-related acute lung injury Article in Blood.

Shander, A. and M.A. Popovsky, 2005. Understanding the consequences of transfusion-related acute lung injury. Chest, 128: 598S-604S. DOI: $10.1378 /$ chest.128.5_suppl_2.598S

Silliman, C.C. and N.J. McLaughlin, 2006. Transfusionrelated acute lung injury. Blood Rev, 20: 139-59. DOI: 10.1016/j.blre.2005.11.001

Silliman, C.C., D.R. Ambruso and L.K. Boshkov, 2005. Transfusion-related acute lung injury. Blood, 105: 2266-2273. DOI: 10.1182/blood-2004-07-2929

Toy, P., O. Gajic, P. Bacchetti, M.R. Looney and M.A. Gropper et al., 2012. Transfusion-related acute lung injury: Incidence and risk factors. Blood, 119: 1757-1767. DOI: 10.1182/blood-2011-08-370932

TTISS, 2012. Summary results for. transfusion transmitted injuries surveillance system.

Vlaar, A.P.J., P. Fung, M. Fung, M.R Looney and N.P. Juffermans et al., 2019. A consensus redefinition of transfusion-related acute lung injury. Transfusion, 59: 2465-2476. DOI: 10.1111/trf.15311

Wallis, J.P., 2003. Tansfusion-Related Acute Lung Injury (TRALI)-- under-diagnosed and under-reported. Br J. Anaesth, 90: 573-576. DOI: 10.1093/bja/aeg101 